\title{
CUTTING MORE THAN METAL: BREAKING THE DEVELOPMENT CYCLE
}

\author{
Chris Singer \\ Director, Engineering \\ NASA Marshall Space Flight Center
}

\begin{abstract}
New technology is changing the way we do business at NASA. The ability to use these new tools is made possible by a learning culture able to embrace innovation, flexibility, and prudent risk tolerance, while retaining the hard-won lessons learned of other successes and failures.

Technologies such as 3-D manufacturing and structured light scanning are reshaping the entire product life cycle, from design and analysis, through production, verification, logistics and operations. New fabrication techniques, verification techniques, integrated analysis, and models that follow the hardware from initial concept through operation are reducing the cost and time of building space hardware.
\end{abstract}

Using these technologies to be more efficient, reliable and affordable requires we bring them to a level safe for NASA systems, maintain appropriate rigor in testing and acceptance, and transition new technology. Maximizing these technologies also requires cultural acceptance and understanding and balancing rules with creativity. Evolved systems engineering processes at NASA are increasingly more flexible than they have been in the past, enabling the implementation of new techniques and approaches.

This paper provides an overview of NASA Marshall Space Flight Center's new approach to development, as well as examples of how that approach has been incorporated into NASA's Space Launch System (SLS) Program, which counts among its key tenants - safety, affordability, and sustainability. One of the 3D technologies that will be discussed in this paper is the design and testing of various rocket engine components. 
The impact of new technology and cultural acceptance is finding its way into NASA's new launch vehicle program, the Space Launch System. The SLS Program is hosted by Marshall, which provides systems engineering and integration, safety and mission assurance, and other important functions. SLS is a super heavy-lift rocket designed for human and robotic missions to deep space. Its initial payload will by NASA's new Orion crew spacecraft. The vehicle is designed to evolve from a 321-foot-tall vehicle capable of launching 70 tons to a 384-foot vehicle that can launch 130 tons. By adopting new technologies and new practices, SLS reached System Requirements Review/Systems Design Review milestones in only 10 months from Program announcement and Preliminary Design Review in two years.

The way SLS employs new technology - often in combination with traditional technology - varies based on the size, complexity, allowable risk, cost, schedule and other factors. Applying these new "art-to-part" 3D modeling technologies in large scale hardware is enabling more integrated design, better fabrication techniques, and better integration of systems and components. In small scale hardware, these technologies permit the development of prototypes, parallel analysis and testing, testing of multiple designs, and proceeding beyond development at significantly lower risk.

Initial experience with additive manufacturing of both new and heritage parts has been dramatic and promising. In several carefully selected applications, the cost savings ranges from 50 to 87 percent, with a time savings of 50 to 80 percent by reducing part count, welds, and machining operations.

Likewise, structured light scanning has allowed SLS to improve process development, reduce cost and schedule through digital assembly, compare small changes in hardware not previously possible, refine performance predictions, reverse engineer heritage parts, and examine subtle details.

Accompanying these new tools is an examination of the NASA culture of perfection and risk aversion. There is a greater understanding of the hardware life cycle and the appropriate balance points in that cycle for flexibility and rigidity. There's a greater emphasis on thinking vs. blind compliance, the need to adjust to program/project size, the importance of integration and communication, and the focus on the product over the process. 